\title{
LAS RECIENTES TRANSFORMACIONES EN IQUIQUE: EL NUEVO SUJETO COSTERO. NORTE DE CHILE
}

\author{
THE RECENT TRANSFORMATIONS IN IQUIQUE: \\ THE NEW COASTAL SUBJECT
}

\author{
Víctor Guerrero Cossio*
}

\begin{abstract}
El presente trabajo da cuenta de la cambiante realidad social costera en la comuna de Iquique, afectada por la expansión económica y el crecimiento poblacional, que en el marco de las nuevas tecnologías produce un nuevo sujeto, transformado en sus relaciones socioespaciales.
\end{abstract}

Palabras claves: Sujetos sociales, desarrollo costero, relaciones socioespaciales, perfil sociocultural.

This essay deals with the ever changing coastal social reality of the commune of Iquique, now influenced by economic expansion and the growth of its population, which insofar as they are connected with today's technologies they produce a new subject, transformed into socio-spatial relations.

Key words: Coastal development, socio-spatial relationships, socio-cultural profile.

\section{Introducción}

La ciudad de Iquique ha crecido vertiginosamente, al punto de no disponer actualmente de terrenos urbanos para la expansión residencial, llevando a ocupar localidades suburbanas y que hasta períodos recientes solo cumplían la función de producción de alimentos para la población. Eso eran Alto Hospicio y las caletas de pescadores hasta la década del 80 del siglo recién pasado.

Alto Hospicio, a partir de 1985, se transformó paulatinamente en una comuna y desde el 2000 el ímpetu de la urbanización se ha trasladado al sector costero, lo que se evidencia en estudios que proyectan un complejo residencial en playas Lobitos, Los Verdes y Tres Islas, que a futuro hacen vislumbrar una nueva comuna en la provincia de Iquique.

La expansión poblacional ha venido acompañada de una mayor complejidad, pues también se han multiplicado las actividades humanas, atingentes a la producción económica y social. Se diversificaron las relaciones sociales y económicas, dando lugar a una verdadera lucha por los recursos del territorio y su transformación en bienes públicos y privados.

Así, el incremento urbano ha ido acompañado de una multiplicación de actividades económicas que también han alcanzado al borde costero sur de Iquique. A diferencia del paisaje costero de antaño, en la actualidad las tradicionales caletas de pescadores son acompañadas de nuevos centros de actividades económicas, como los puertos mineros, aeropuerto internacional, centros de recreación y restaurantes, además de un explosivo aumento del tránsito de vehículos por la ruta costera. Estas nuevas actividades han aumentado la artificialización en la naturaleza costera, sin embargo lo más reciente y amenazador es la inminente instalación de centrales de energía termoeléctricas.

En este sentido hay que considerar que actualmente los recursos del espacio costero no se limitan a la capacidad de recursos pesqueros para la extracción artesanal, sino que se amplían hacia riquezas que anteriormente no se advertían en su potencialidad y usos.

El paisaje costero, la temperatura del mar, la pradera marina y otros aspectos antiguos y emergentes, son una multivariada realidad, objeto de apropiación de parte de diversos actores económicos.

Es menester dar cuenta de esta nueva producción del espacio social en Iquique, trascendiendo el hábitat urbano de la ciudad, pues la costa está ofreciendo un escenario que desde hace una década asienta a diversos actores que protagonizan una disputa por sus áreas de mayor riqueza. Hoy esa realidad económica es contradictoria, los usos de unas perturban los fines de otras.

Las contradicciones de este proceso de apropiación del territorio han generado tensiones conducentes a negociaciones, movilizaciones y lobby, a fin de ganar ventajas en el acceso al territorio. $\mathrm{La}$

\footnotetext{
* Universidad Arturo Prat, Iquique, Chile. Correo electrónico: victorguerrerocossio@gmail.com
} 
compleja nueva realidad costera no puede dejarse al espontaneísmo y menos al mercado la asignación total de sus recursos, porque el patrimonio cultural, los derechos sociales y la preservación del ambiente, necesarios para una economía sustentable, requieren una adecuada planificación por parte del Estado, quien debe hacerse cargo de los efectos del nuevo Iquique.

El presente trabajo centrará el análisis en los asentamientos de pescadores artesanales, para que en función de su transformación se puedan advertir las tensiones existentes en el borde costero, las que pueden llevar a cuadros insostenibles en las relaciones sociales, económicas y ambientales. Hoy se puede hablar de la transformación territorial en términos materiales, pero también de la emergencia de un nuevo sujeto social, como producto de estas nuevas condiciones imperantes.

\section{Breve caracterización poblacional de la Región de Tarapacá}

La Región de Tarapacá limita al oeste con el océano Pacífico, al este con la república de Bolivia, al norte con la Región de Arica y Parinacota y al sur con la Región de Antofagasta. La Región de Tarapacá tiene una superficie de $42.225,8 \mathrm{~km}^{2}$, con una población de 298.257 habitantes. Su densidad poblacional es de 5,65 habitantes / $\mathrm{km}^{2}$.

La capital regional es Iquique y cuenta con 238.950 habitantes, concentrando al 90,6\% de la población total de Tarapacá. Las siguientes ciudades más pobladas son Pozo Almonte y Pica, con 10.830 y 6.178 habitantes, respectivamente (Censo 2012). (Tabla 1 y 2).

La información que surge de estos antecedentes generales es el notable incremento poblacional ocurrido en la década del 90, la que se tendió a estabilizar en la década siguiente. No obstante, la región más que duplicó su población entre 1982 y 2012, aumentando las necesidades de ocupación de territorio y actividad económica.

Lo señalado llevó a que desde los 90 se iniciara la creación de la comuna de Alto Hospicio, por la propia necesidad residencial y los costos crecientes de $\mathrm{m}^{2}$ de terreno urbano, mientras que en los inicios del siglo XXI ocurre un fenómeno semejante, esta vez afectando a la zona costera sur de Iquique, para fines económicos y habitacionales.

\section{Caracterización del Borde Costero sur de Iquique}

El borde costero en la región de Tarapacá involucra a las comunas de Iquique, litoral sur, y la comuna de Huara, litoral norte. Pese a esta ubicación geográfica el área costera referida es una extensión que abarca la totalidad del territorio regional y que conforma una extensión de 241 kilómetros entre Pisagua y El Loa.

En la actualidad este espacio ha cambiado su fisonomía debido a la creciente y diversificada acti-vidad económica, la que suma a los asentamientos tradicionales -ligados a la pesca artesanal- un con-junto de asentamientos modernos, tanto productivos como de servicios.

Tabla 1. Población en la Región de Tarapacá

\begin{tabular}{|c|c|c|c|c|c|c|c|}
\hline \multirow[b]{2}{*}{ Región } & \multicolumn{4}{|c|}{ Censo } & \multicolumn{3}{|c|}{ Variación } \\
\hline & 1982 & 1992 & 2002 & 2012 & $82-92$ & $92-02$ & $02-12$ \\
\hline I de Tarapacá & 122.729 & 163.404 & 236.021 & 298.257 & 33,1 & 44,4 & 26,4 \\
\hline
\end{tabular}

Fuente: Censo Nacional 1982, 1992, 2002, 2012. Instituto Nacional de Estadísticas.

Tabla 2. Población Rural-Urbana

\begin{tabular}{ccccccc}
\hline Año & Total & Hombre & Mujer & Rural & Urbano & $\%$ \\
\hline 2012 & 300.021 & 151.084 & 148.937 & 20.613 & 279.408 & 7,37 \\
2002 & 238.950 & 123.072 & 115.878 & 12.488 & 226.460 & 5,51 \\
1992 & 165.469 & 83.995 & 81.474 & 9.071 & 156.389 & 5,80 \\
\hline
\end{tabular}

Fuente: Censo Nacional 1992, 2002, 2012. Instituto Nacional de Estadísticas. 
Tabla 3. Población residente en asentamientos pesqueros artesanales

\begin{tabular}{|c|c|c|c|c|}
\hline Asentamiento humano & Tipo & Comuna & Clasificación & Hab. \\
\hline Tres Islas & Localidad & Iquique & Rural & 20 \\
\hline Playa Blanca & Localidad & Iquique & Rural & 60 \\
\hline Punta Gruesa & Localidad & Iquique & Rural & 5 \\
\hline Los Verdes & Caleta & Iquique & Rural & 248 \\
\hline Playa Pozo Toyo & Localidad & Iquique & Rural & 49 \\
\hline Caramucho & Localidad & Iquique & Rural & 81 \\
\hline Yape & Localidad & Iquique & Rural & 8 \\
\hline Caleta Chanavayita & Caleta & Iquique & Rural & 1.434 \\
\hline Patillo & Localidad & Iquique & Rural & 8 \\
\hline Cáñamo & Localidad & Iquique & Rural & 260 \\
\hline Caleta Chanavaya & Caleta & Iquique & Rural & 81 \\
\hline Río Seco & Localidad & Iquique & Rural & 150 \\
\hline Caleta San Marcos & Caleta & Iquique & Rural & 241 \\
\hline Guanillos & Localidad & Iquique & Rural & 0 \\
\hline Caleta Chipana & Caleta & Iquique & Rural & 179 \\
\hline Río Loa & Localidad & Iquique & Rural & 13 \\
\hline Total & & & & 2.837 \\
\hline
\end{tabular}

Fuente: CENSO 2002 y actualización Municipio Iquique 2015.

Según la información precedente (tabla 3), Chanavayita es la localidad rural y caleta pesquera de mayor importancia, refrendada como tal al ser exclusiva-mente considerada como Aldea en el litoral, según el Instituto Nacional de Estadísticas.

De 1.292 habitantes registrados en el censo del 2002, se ha incrementado a 2.837 en la actualidad, de acuerdo con estudios realizados por organismos municipales.

También cobran creciente importancia los terminales portuarios de Patillos y Patache, asimismo el aeropuerto Diego Aracena, que han incrementado considerablemente su población laboral y que en muchos casos han decidido radicarse en caletas de pescadores aledañas, como Cáñamo, Chanavallita y Río Seco. Estos son decisivos actores de la nueva realidad costera, por la cantidad de acciones económicas, número de trabajadores que operan y se desplazan diariamente en el área, también por el impacto ambiental que generan.

La población minera, portuaria y de servicios no reside en sus establecimientos, pero concurren diariamente a ellos, estableciendo así una relación permanente en dichos lugares. Sin embargo en los últimos años parte de esta población ha optado por residir en caletas de pescadores, anteriormente habitadas solo por estos.

El desarrollo del borde costero presenta cada vez más actores que compiten por el uso de los recursos de este territorio, aumentando la complejidad, las demandas y los conflictos sociales y económicos en el sector. Es menester observar esta dinámica social con una perspectiva moderna de la geografía, más centrada en la dinámica que en la estática, más en lo social que en lo físico.

Se puede decir que la geografía se interesó más por la forma de las cosas que por su formación. Su dominio no era el de las dinámicas sociales que crean y transforman las formas, sino el de las cosas ya cristalizadas. Esto proporciona una imagen invertida que impide aprehender la realidad si no se hace intervenir la historia. Si la geografía desea interpretar el espacio humano como el hecho his-tórico que es, solamente la historia de la sociedad mundial, aliada a la sociedad local, puede servir como fundamento a la comprensión de la realidad espacial y permitir su transformación al servicio del hombre. La historia no se escribe fuera del espacio y no hay sociedad aespacial. El espacio es, en sí mismo, social (Santos et al. 1996: 16).

El Estado chileno ha respondido a estos mayores requerimientos con una atención creciente, prin-cipalmente de los ministerios y servicios públicos relacionados. En particular debe mencionarse el trabajo de zonificación costera que ha impulsado la Subsecretaría de Desarrollo regional, los estudios concernientes a habitabilidad y expansión urbana del MINVU, los estudios y ejecución de proyectos del MOP. Estos trabajos se encuentran en la base del presente informe, incluyendo también información propia. 
La envergadura de los problemas que se han presentado en los últimos años ha evidenciado la baja capacidad de reacción mostrada por algunas entidades públicas, especialmente el Ministerio de Medio Ambiente frente a contingencias importantes, como la instalación de termoeléctricas por parte de la empresa privada. En este sentido también debe señalarse la baja capacidad que ha tenido la Dirección Regional de Turismo para entregar soportes adecuados a los proyectos turísticos, que también se ven amenazados por las tensiones producidas en este aumento de demanda por la apropiación de territorio ubicado en el borde costero.

"Ninguna sociedad tiene funciones permanentes, ni un nivel de fuerzas productivas fijo, ninguna está marcada por formas definitivas de propiedad, de relaciones sociales". "Etapas en el transcurso de un proceso", como Labriola las definió, la Formación Económica Social no puede ser comprendida sino en el marco de un movimiento totalizado, en el que todos sus elementos son variables que interactúan y evolucionan juntos, sometidos a la ley del todo (Santos et al. 1996: 20).

Este panorama revela la expansión del interés económico en el borde costero de Tarapacá, augurando un crecimiento de las tensiones, tanto sociales como económicas y tecnológicas. En los próximos años el desarrollo del sector demandará más provisión energética, hídrica, conectividad y habitabilidad, lo que requerirá interacciones importantes del sector público con el privado, tanto a nivel de empresas como de grupos sociales organizados, especialmente pescadores artesanales y juntas de vecinos.

\section{La nueva realidad costera: problemas y conflictos}

La zona costera sur de Iquique presenta distintas características, problemas y conflictos debido a la diferente densidad económica y social registrada en los últimos veinte años.

En el litoral sur de Iquique, conformado por varios asentamientos humanos de orden costero, existe una diversidad de actividades económicas y sociales, las que crecientemente comienzan a desatar tensiones por el usufructo de la naturaleza. Las hay de orden pesquero artesanal (caletas de pescadores), portuarias para embarque de minería metálica y no metálica (sal y cobre), transporte aéreo (aeropuerto Diego Aracena), industriales (minería y pesca) y turismo (restaurantes y centros recreativos).
Esta nueva realidad social ha conllevado un cambio en las relaciones entre los sujetos, avanzando hacia una mayor diversidad e intensidad debido a la multiplicación de intereses sociales y acciones económicas.

Aunque se analizó la relación entre sujetos y objetos que circulan cada vez más rápido, más aún, se trabajó acerca de la relación recíproca entre sujetos comprometidos en una circulación siempre más veloz. Simmel contrapuso esas interacciones sujeto-sujeto de la modernidad a las que se conocían en las sociedades tradicionales. En la modernidad, las interacciones son pasajeras, intensas y diversas (con muchos y muy diferentes otros), mientras que en las sociedades tradicionales son de larga duración, difusas y uniformes (se hacen con otros que son pocos y muy similares (Lash y Urry 1998: 30).

Los asentamientos humanos relevantes son más numerosos y diversos que hace diez años, alcanzando a 15 centros poblados, sin embargo en esta presentación se enfatizan aquellos de mayor connotación y donde se ha concentrado la mayor dinámica. Para el caso de la actividad pesquera, residencial y comercial estos son los siguientes:

\section{Descripción socioeconómica y productiva del borde costero de la Región de Tarapacá}

Se advierte mayor cantidad y complejidad de las relaciones de la población que habita en el borde costero, tanto en lo económico como en lo social, a su vez las demandas al Estado aumentan y se diversifican. Las tensiones entre agentes económicos y sociales aumentan, en una verdadera disputa por el mar, en su sentido productivo, de transporte y paisaje, como la utilización de las zonas de playa que también son demandadas para fines divergentes.

La aparición de nuevos temas sociales, como también la constitución de Juntas de Vecinos y dirigentes de género femenino como líderes de ellas, permiten advertir la transformación de la realidad costera. Es importante indicar también que los conflictos no solo son entre los trabajadores del mar y los nuevos trabajadores de la costa sur de Iquique, sino entre pescadores artesanales y algueros que desarrollan una actividad depredadora de la pradera marina.

Según el estudio "Diagnóstico macrozonificación de usos del borde costero", el área costera sur de Iquique, desde Bajo Molle hasta El Loa, frontera con la región de Antofagasta, muestra con claridad 
las proyecciones de la zona costera y revela que la tendencia a diversificar las actividades, exacerbando las tensiones por la disputa del uso del espacio geográfico, se incrementará en los próximos años.

Este documento clasifica tres áreas, en las que destacan actividades emergentes que se suman a las tradicionales, como:

- Hasta Playa Quinteros: puertos, pesca artesanal, comercio, turismo, hostales y restaurantes. Se incorpora también la función humana-demográfica, mediante el uso residencial urbano y rural, recreacional en gran parte de la costa urbana, y por último la función de infraestructura de tipo público, vial y marítimo portuario.

- Hasta Punta Lobos. Posee una función productiva esencialmente industrial portuaria, en la que se desembarcan minerales procesados en lo alto de la comuna de Iquique y de la comuna de Pica. Camiones cargados de sal se enlazan finalmente con puerto de Patillos, y en un futuro cercano con Patache, para su envío a destino internacional. Por otro lado, los concentrados de cobre son enviados por medio de un mineroducto que conecta la faena minera de Collahuasi con sus instalaciones portuarias, asimismo ascienden camiones de ácido sulfúrico por la ruta de la sal.

- Hasta el límite con la Región de Antofagasta. Alberga un uso eminentemente pesquero artesanal con algunas concesiones acuícolas. Esta franja se encuentra cubierta por un gran número de concesiones mineras en exploración, que se posicionan entre dos caletas pesqueras importantes para el borde costero (caleta San Marcos y Chipana).

En este territorio, tradicionalmente ocupado por la actividad pesquera artesanal, se mantienen ciertos problemas de redes de conexión sanitaria y social, a los que se suman las contradicciones con las actividades modernas, más ligadas a la minería y a los servicios de movilidad y esparcimiento asociados a la actividad urbana.

\section{Los impactos sociales en los pescadores artesanales}

La nueva realidad del borde costero sur ha tenido impactos especialmente en los más antiguos actores sociales, como son los pescadores artesanales; los nuevos trabajadores sufren recién su proceso de adaptación al medio y su relación con este es diferente, de menor presencia, intermitente, espasmódica.

Las nuevas prácticas sociales derivadas de la transformación del espacio social, en un contexto de tensiones socioproductivas, de riesgos ecosociales y de nuevas expectativas socioeconómicas, han transformado las características del sujeto social costero no solo por la presencia de los nuevos actores, sino por una simbiosis ocurrida en los antiguos pescadores artesanales a causa de su nueva y más compleja red de interrelaciones sociales.

"Problemas harto difíciles de resolver, debido a que cada vez está menos claro lo que se entiende por esta disciplina o por lo rural como objeto de estudio de ella en una sociedad mundializada en la que, a medida que se incrementa el nivel de extensión de las pautas urbano-industriales, tienden a tornarse más difusas las características y las fronteras de lo rural" (Entrena Durán 1998: 11).

Asimismo, es menester considerar las nuevas relaciones que se establecen entre los habitantes de la ciudad -Iquique- y de la costa -pescadores, portuarios, oficinistas-, quienes concurren diariamente a sus actividades laborales, a diferencia de la población tradicional que se mantenía en sus asentamientos humanos con mayor permanencia.

"Lo rural se construye socialmente y como construcción social está sujeto al cambio. Como consecuencia del cambio suelen experimentarse sucesivos procesos de construcción, deconstrucción o reconstrucción/reinvención de lo rural. El considerable grado de variabilidad de los ámbitos espacio-temporales en los que pueden experimentarse tales procesos, así como la diversidad se acciones y presiones colectivas que eventualmente pueden condicionar los resultados de los mismos, determina el carácter mutante y circunstancial de lo rural, cuya forma de análisis más adecuada es, por tanto, la que adopta perspectivas histórico-procesuales" (Entrena Durán 1998: 14).

\section{Perfil de pescadores artesanales}

Un trabajo publicado en 1998, "Transformaciones en los asentamientos rurales: el caso de Iquique" (Última Década, 1998, pp. 4-5), daba cuenta del perfil social que registraban los pescadores artesanales hace veinte años. En el presente artículo se ha querido actualizar este perfil, el que indica cambios relacionados con aumento de aportes estatales y 
mayor vinculación a los centros urbanos más grande, aunque contradictoriamente dicha transformación no se expresa en un mejor desarrollo productivo, pero sí en la realidad social de los asentamientos, haciendo de estos escenarios de superior complejidad.

\section{Nuevo perfil artesanal pesquero}

La realidad actual de las localidades costeras, transformada por las nuevas actividades económicas, por flujos laborales y residenciales, pero también por la intervención estatal y la influencia de un mundo globalizado, ha generado un contexto que propicia un cuadro nuevo de relaciones sociales y la generación de condiciones que varían el perfil del pescador artesanal.

\section{a. Migración estacional}

La población artesanal pesquera ha disminuido sus anteriores largas migraciones, tendiéndose a su establecimiento en las caletas o bien manteniendo viviendas en la ciudad de Iquique, desde donde se desplazan diaria o periódicamente a los centros de extracción.

Las nuevas condiciones materiales y acceso tecnológico permiten que los pescadores generen mejores condiciones de habitabilidad y bienestar familiar, permitiendo un asentamiento más arraigado en la localidad.

\section{b. Dependencia económica}

Su relación asimétrica en el mercadeo que establecen con compradores mayoristas no ha variado sustancialmente, persistiendo su carácter dependiente. Es la conclusión a la que llegan la mayoría de los estudios en esta área, existiendo cambios significativos principalmente en los medios de transporte de los antiguos rematadores, hoy convertidos en medianos empresarios. Estos sí mejoran sus condiciones de trabajo, preservación de la producción y conexiones financieras con la banca local.

\section{c. Marginalidad social}

Sus estrategias de sobrevivencia y aproximación al mundo global han variado significativamente, en especial por el acceso a la red estatal y a las tecnologías de la comunicación. Han aumentado los niveles de educación familiar por la maduración de los proyectos educativos municipales y a la vez mejorado el acceso a la previsión social. Su falta de independencia comercial y baja inversión en las artes de pesca no ha reducido significativamente la inestabilidad salarial y escasa seguridad laboral.

\section{d. Problemas sociales}

Aún destacan los cuadros de alcoholismo y drogadicción, acentuándose estos últimos por la presencia cada vez mayor del tráfico de estupefacientes en la región. A la vez se mantienen cuadros importantes de violencia intra y extrafamiliar, pero la red estatal aporta protecciones sociales relevantes en esta materia, haciendo que las familias aumenten su protección. Ha disminuido notoriamente la deserción e inestabilidad escolar, así como la falta de medios y baja vinculación con el mundo externo.

\section{e. Concepción del mundo}

Persiste el énfasis en la realidad inmediata, pero con acceso a las redes sociotecnológicas y con ello vinculación al mundo externo, aumentando y diversificando sus expectativas como sujeto del siglo XXI. Esto ha permitido aumentar sus capacidades de abstracción, perspectivas, planes y expectativas, incrementando por ello sus capacidades de imaginar mundos futuros distintos.

La nueva realidad social, la competencia por los recursos, el contexto globalizado y la red estatal, han mejorado y ampliado su vida cotidiana, permitiendo a estos sujetos trascender el "día a día" y moderar su desconfianza respecto de los beneficios que no sean individuales e inmediatos.

Su ética instrumental ha evolucionado a un carácter más complejo, conducida por mayores niveles de competencia y sustentabilidad en su sector, promovidas por el mercado y el Estado, respectivamente. Este mayor panorama y visión trascendente de los beneficios individuales, directos e inmediatos, ha mejorado en estos sujetos la comprensión y adhesión a cuadros más complejos, mediatos, indirectos y colectivos, de la gratificación social.

\section{f. Tecnología y ethos laboral}

Mayor apertura a la introducción de técnicas y modos de producir. Contradicción entre su perspectiva respecto de temas ecológicos. 
Han facilitado su sensibilidad ambiental las amenazas de competidores en el medio costero, como los depredadores de la flora marina (extractores de huiro), la mayor temperatura del mar en las zonas inmediatas a los puertos e instalaciones mineras costeras (mineroducto de Cía. Minera Collahuasi), experiencias en desarrollo sustentable (cultivos de ostras y ostiones) y exigencias ambientales estatales en cuanto a la preservación de los recursos (áreas de manejo).

La razón ambiental contradictoria que mantiene la inhibición ecológica en sus prácticas reside en la mantención de limitaciones económicas y tecnológicas para asegurar su sobrevivencia, especialmente las trabas burocráticas (vedas), variaciones climáticas (marejadas) y de mercado (bajos precios).

\section{g. Perfil ocupacional}

Hay presencia de una mayor estabilidad y antigüedad ocupacional en el área, lo que redunda en una mayor consolidación de sus prácticas laborales y por tal razón de una producción de su identidad cultural. Poblaciones humanas provenientes de otros espacios ocupacionales, atraídos al sector por desempleo u otro cese de labores en la ocupación original, se han asentado y convertido en trabajadores del mar.

En el marco del nuevo contexto existente en el borde costero las poblaciones laborales han producido un sistema precario de costumbres, valores y proyecto colectivo acorde a la sociedad del siglo XXI, con sus virtudes y defectos. Hay una nueva estructura social, considerando esta principalmente como la materialización de una pauta de conducta esperable y legitimada por las acciones prácticas.

\section{Conclusiones}

Las tendencias actuales en el borde costero sur de Iquique, debido a las recientes dinámicas promovidas por la revolución económica y tecnológica que imperan en el mundo en las últimas décadas, ha imprimido en los sujetos que viven en la costa y de sus recursos, tanto de las actividades tradicionales como de las nuevas, un conjunto de expectativas y tendencias culturales que han transformado notablemente su realidad social.

Se han constituido en un nuevo sujeto y el reconocimiento de sus actuales características es necesario para comprender sus conductas frente a sus necesidades y priorizaciones, que muestran un panorama económico de mayor movilidad y diferenciación, que aumenta la competencia entre sus habitantes y deprime la cohesión sociocultural.

Esta nueva realidad, consistente con la cristalización de un nuevo sujeto social, muestra una mayor complejidad de los asentamientos humanos costeros, caracterizados por la reestructuración de sus patrones de conducta, la variación de sus componentes poblacionales y la pronta variación de sus hábitos culturales.

Un elemento contradictorio es el hecho de que junto a la menor perdurabilidad de sus estructuras se advierte una mutación incesante de sus contenidos, lo que es impulsado por las características de menor apego y solidez, líquidas, al decir del sociólogo Bauman.

La envergadura y profundidad de los procesos señalados requiere atención sistemática para enfrentar los conflictos y evitar que estos encuentren soluciones espontáneas, los que normalmente afectan de manera negativa a los actores sociales más débiles.

Nos guste o no, por acción u omisión, todos estamos en movimiento. Lo estamos aunque físicamente permanezcamos en reposo: la inmovilidad no es una opción realista en un mundo de cambio permanente. Sin embargo, los efectos de la nueva condición son drásticamente desiguales. Algunos nos volvemos plena y verdaderamente "globales"; otros quedan detenidos en su "localidad", un trance que no resulta agradable ni soportable en un mundo en que los "globales" dan el tono e imponen las reglas del juego de la vida.

Ser local en un mundo globalizado es una señal de penuria y degradación social. Las desventajas de la existencia localizada se ven acentuadas por el hecho de que los espacios públicos se hallan fuera de su alcance, con ello las localidades pierden su capacidad de generar y negociar valor. Así, dependen cada vez más de acciones que otorgan e interpretan valor, sobre las cuales no ejercen el menor control.

Los procesos globalizadores incluyen una segregación, separación y marginación social progresiva. Con la libre movilidad en su centro, la polarización actual tiene muchas dimensiones. Este nuevo centro da nuevo lustre a las distinciones consagradas entre ricos y pobres; nómadas y sedentarios; lo "normal" y lo anormal, y lo que está dentro o fuera de la ley (Bauman 1996: 56).

En términos prácticos, quizás en el nuevo contexto las demandas sociales más relevantes son aquellas 
relacionadas con los derechos sociales y ambientales, debido a la inminencia de que se agudicen las tensiones entre los actores que se disputan los recursos naturales del espacio costero. En la actualidad, como se señaló anteriormente, la riqueza para unos afecta y se transforma en pobreza para otros.

Destacan en estas nuevas luchas por la apropiación del medio la actividad pesquera artesanal y asentamientos humanos asociados, la proyección inmobiliaria derivada de la expansión urbana de Iquique, el turismo masivo y selectivo, las obras portuarias y energéticas, el incremento del transporte urbano. Ante ello, urge la planificación del desarrollo costero, a fin de alcanzar uno de carácter sustentable.

Las proyecciones estimadas para el sector, desde el punto de vista de los estudios realizados por instituciones públicas, no han considerado de manera suficiente las características del sector, las que presionan por un modelo de desarrollo sustentable. El equilibrio natural es precario y las demandas que se advierten pueden llevar a una crisis de los recursos y atributos en que descansa su potencialidad. Al respecto no se debe olvidar que estos espacios, que presentan ecosistemas originales, son altamente dinámicos y frágiles en sus equilibrios. Presentan grandes potencialidades de desarrollo, pero también tienen limitaciones de uso e implican riesgos que se deben tomar seriamente en cuenta si se desea mantener su paisaje y su biodiversidad, para heredarlos sin deterioro irreversible a las próximas generaciones.

El nuevo sujeto costero de la comuna de Iquique es producto de esta nueva realidad social, multifacética, cambiante y más compleja. Que gradualmente presenta demandas que presionan por una mayor estabilidad en sus asentamientos, los que contradictoriamente crecen en la misma medida que otros actores económicos - mineros, turísticos y comerciales-, como también nuevas explotaciones artesanales -extracciones de huiros-que compiten en el uso de los recursos ambientales.

\section{Referencias Citadas}

\footnotetext{
Bauman, Zigmunt

1996 La Globalización: consecuencias humanas. Fondo de Cultura Económica, México.

Entrena Durán, Francisco

1998 Cambios en la Construcción Social de lo Rural. De la autarquía a la globalización. Ed. Tecnos, España.

Instituto Nacional de Estadísticas (INE). Censo 2012.

Lash, Scott y Urry, John

1998 Economía de signos y espacios. Amorrortu Editores.

B. Aires.
}

Santos, Milton y Martínez Rigol, Sergi 1996 De la Totalidad al lugar. Ed. Oikos-tau. España.

Santos, Milton y Martínez Rigol, Sergi 1996 Metamorfosis del Espacio Habitado. Ed. Oikos-tau, España.

SUBDERE, Gobierno de Chile.

2011 Diagnóstico macrozonificación de usos del borde costero. 